\title{
LE NUCLÉAIRE ET LE DROIT
}

\section{Présentation}

$D$ ans le cadre des activités d'information et de concertation entreprises par la Société Française d'Energie Nucléaire, la Section Technique «Contextes Energétique, Economique et Financier»a apporté son concours à l'Ecole Nationale de la Magistrature (ENM) de Bordeaux pour organiser à Grenoble, les 11 et 12 décembre 1980, une session de Formation Permanente de deux jours sur le thème «Le Nucléaire et le Droit».

Le programme de cette manifestation et le choix des conférenciers ont été faits en étroite liaison avec M. Espel, Maître de Conférences à l'ENM de Bordeaux, et avec le Comité des Relations Publiques de la SFEN.

La première partie de cette session a été constituée par trois communications destinées à présenter le nucléaire et à rappeler aux participants un certain nombre de données permettant de situer le contexte $d u$ «fait nucléaire» et de mieux appréhender les divers problèmes qui en découlent.

Ces trois communications, à savoir :

- «Présentation technique simplifiée », par M. Roquefort (EDF REAL);

- «Le programme français et son économie», par $M$. Hammer (CEA);

- «L'industrie nucléaire française et les aspects financiers du programme français », par M. Grau (Framatome),

portent sur des sujets déjà traités dans la RGN. Pour cette raison, la rédaction de la revue n'a pas jugé nécessaire de les publier.
Par contre, il lui a semblé intéressant de publier «in extenso », dans la présente livraison de la RGN, le texte des communications qui traitent plus spécifiquement des relations entre «le Nucléaire et le Droit», relations qui constituaient l'objet fondamental de la session.

Les diverses questions évoquées durant ces deux journées ont soulevé un très vif intérêt dans l'auditoire: des débats très animés ont prouvé, s'il en était besoin, la complexité des problèmes soulevés et la variété de leur perception.

P. Grau,

Président de la Section Contextes Energétique, Economique et Financier de la SFEN.

La RGN tient à remercier MM. Espel et Grau pour la part qu'ils ont prise à la réalisation de ce dossier.

90 / RGN - 1981 - № 2 - Mars-Avril 